\title{
Chapter 16 \\ An Overview of the CFD Analyses in the MARE-WINT Project
}

\author{
George N. Barakos
}

\begin{abstract}
In the MARE-WINT project, two early stage researchers, researched and developed CFD methods for complete offshore wind turbine configurations. This brief chapter provides an overview of the work conducted by these two fellows.
\end{abstract}

The subsequent two chapters detail the work carried out during the MARE-WINT project on the CFD simulation of complete wind turbines. The term 'complete' needs to be clearly defined, since it is used here only in relation to the current CFD state-of-the-art. Often, Wind Turbine (WT) blades are analysed using grid-based Navier-Stokes methods under the assumption of steady flow with spatial symmetry between blades. The inflow to the blades is uniform, the tower, spinner and nacelle are typically ignored and the aeroelastic effects are absent.

The work at MARE-WINT progressed beyond the current state-of-the art by presenting results for configurations that include all blades, the tower and nacelle, aeroelasticity and even the effect of the wind turbine motion due to waves for floating off-shore configurations. An additional feature of the current work is that large scale wind turbines are considered in contrast to past CFD works that mainly simulated scaled wind turbines used for validation of CFD against wind tunnel data.

Two fellows contributed to this task, and one common theme between their works is that they both used compressible CFD solvers. This was driven by two reasons. On one hand, the solvers were readily available and well-validated for high speed aerodynamic flows (compressible flows) like flows around transonic aerofoils, cascades, helicopter rotors etc. The methods used low-Mach and all-Mach schemes to account for the relatively low speed flow around wind turbines. Both methods used multi-block structured grids with sliding grids to account for the relative motion between blades and the tower. The solvers used turbulence models of the $\mathrm{k}-\omega$ family for their work. The use of structured grids was combined with the use of aeroelastic methods. These fall within the established Fluid/Structure Interaction methods that are common in aerospace applications. The methods include not only modal-based representations of the blades but also mesh deformation, interpolation

\footnotetext{
G.N. Barakos $(\bowtie)$

Division of Aerospace Sciences, School of Engineering, University of Glasgow, James Watt South Building, Glasgow G12 8QQ, UK

e-mail: george.barakos@glasgow.ac.uk
} 
and motion methods to accommodate the dynamic change of the blade shape during its motion.

One of the researchers went even further by considering flaps on the blades as described in other paragraphs of this book. Furthermore, the CFD methods were coupled with a hydrodynamic method to allow for the simultaneous solution of the liquid domain near the water-surface of off-shore cases. Additional modelling elements worth of mention here, include the use of a multi-body dynamics tool to allow for the effect of mooring lines and the overall rigid motion of the complex WT system to be accounted for effectively. The use of multi-body simulation is common in the context of simpler engineering methods than CFD, where the bladeelement momentum theory is used to estimate the blade loads but it has so far been unexplored for grid-based Navier-Stokes methods.

To close the introduction to this part it should be mentioned that more elaborate models of complete wind turbines would include a time-varying inflow as well as a model of the drive-train and the control system of the WT. These are extensions that should be attempted by the fellows of MARE-WINT in the future as their work progresses and their skills are advancing. Needless to mention here that the CFD analyses of the complete wind turbines are to benefit from parallel computing and especially the use of new processor technologies like Graphical Processing Units as well as many-core machines like the Intel phil.

Extrapolating from this work, one expects to see in the future, work on extreme and fatigue loads with CFD replacing some of the BEM methods that are now used due to the large number of cases necessary to be computed for the overall loads envelope of a WT. Additional applications will include active rotors with flaps and flow control systems, as well as, direct simulation of wind turbine noise. Farm analysis is also expected to move away from the free-wake and actuator disk LESlike simulations, to properly resolved blades with high order schemes able to capture and maintain wakes for the necessary length of time and space.

Open Access This chapter is distributed under the terms of the Creative Commons AttributionNonCommercial 4.0 International License (http://creativecommons.org/licenses/by-nc/4.0/), which permits any noncommercial use, duplication, adaptation, distribution and reproduction in any medium or format, as long as you give appropriate credit to the original author(s) and the source, provide a link to the Creative Commons license and indicate if changes were made.

The images or other third party material in this chapter are included in the work's Creative Commons license, unless indicated otherwise in the credit line; if such material is not included in the work's Creative Commons license and the respective action is not permitted by statutory regulation, users will need to obtain permission from the license holder to duplicate, adapt or reproduce the material. 